\title{
A resistivity model for electrophoretic deposition
}

\author{
Begoña Ferrari ${ }^{1, a}$, Rodrigo Moreno ${ }^{1, b}$, Jose A. Cuesta ${ }^{2, c}$ \\ ${ }^{1}$ Instituto de Cerámica y Vidrio, CSIC, c/ Kelsen, nº 5, Cantoblanco, Madrid, E28049, Spain \\ ${ }^{2}$ Grupo Interdisciplinar de Sistemas Complejos (GISC), Dpto. Matemáticas, UC3, Leganés, \\ E28911, Spain \\ abferrari@icv.csic.es, ${ }^{b}$ rmoreno@icv.csic.es, ${ }^{c}$ cuesta@math.uc3m.es
}

Keywords: EPD kinetics, EPD modelling

\begin{abstract}
Electrophoretic deposition (EPD) is a powerful method for obtaining particulate layers in a broad range of thicknesses if an adequate control of the growing kinetics is reached. Existing models of EPD kinetics consider that the growth of the deposit increases linearly with deposition time and deviations are due to a reduction of powder concentration and/or a decrease of electric field when EPD is performed at constant voltage conditions. Experimental observations show that long time tests lead to a S-shaped growing kinetics. This work presents a resistivity model that predicts a S-shape variation of mass per unit area with deposition time, with a first step in which the deposition rate increases, as a consequence of resistivity changes, followed by a decreasing slope associated to the lose of powder concentration. Currently available EPD models, such as the Hamaker and Sarkar \& Nicholson models are particular cases of the generalized resistivity model proposed in this work.
\end{abstract}

\section{Introduction}

The most basic model of EPD kinetics is that proposed by Hamaker (Eq. 1), which relates the deposited mass per unit area, $\mathrm{m}(\mathrm{g})$, with slurry properties, such as concentration, $\mathrm{C}\left(\mathrm{g} \mathrm{cm}^{-3}\right)$, and electrophoretic mobility, $\mathrm{u}_{\mathrm{e}}\left(\mathrm{cm}^{2} \mathrm{~s}^{-1} \mathrm{~V}^{-1}\right)$, and with the physical and electrical conditions imposed on the system: electric field, $\mathrm{E}\left(\mathrm{V} \mathrm{cm}{ }^{-1}\right)$, deposition area, $\mathrm{S}\left(\mathrm{cm}^{2}\right)$, and deposition time, $\mathrm{t}(\mathrm{s})[1]$,

$m=C u_{e} S E t$

The linear variation of deposited mass with deposition time requires that the parameters of equation 1 remain unchanged with time. Hence, the Hamaker equation is only valid for short times.

The electric field in the bulk of a homogenous suspension, without ions or particles concentration gradients, is related to the applied current density, $\mathrm{i},\left(\mathrm{A} \mathrm{cm}^{-2}\right)$ by:

$E=i \rho$.

$\rho(\Omega \mathrm{cm})$ being the resistivity of the suspension. Then, Eq. 1 can be rewritten as,

$m=u_{e} i \rho S C t$.

If the conduction surface and the area for deposition do not vary with time eq. 1 can be expressed in terms of the applied current intensity, I (A), in the form:

$m=u_{e} I \rho C t$

Sarkar and Nicholson [2] analyzed the dependence of kinetics with experimental EPD conditions such as the influence of electrical parameters or the variation of powder concentration. The validity of this relationship requires the applied current density and the solid concentration to be constant. 
At the earlier stages of the process the variation of solid concentration in the bulk is negligible since only a minor fraction of powder is being deposited. Hence, for infinitesimal intervals of time the Hamaker equation always holds, so that

$$
\frac{d m}{d t}=u_{e} I \rho C
$$

For longer times the amount of powder that is excluded from the bulk suspension may become significant and hence $\mathrm{C}$ decreases. The deposited mass and the solid content are related by:

$$
m=V\left(C_{0}-C\right)=\frac{M}{C_{0}}\left(C_{0}-C\right)
$$

where $\mathrm{C}_{0}\left(\mathrm{~g} \mathrm{~cm}^{-3}\right)$ is the initial solid content of the suspension, $\mathrm{V}\left(\mathrm{cm}^{-3}\right)$ is the volume of suspension and $\mathrm{M}(\mathrm{g})$ is the initial mass of powder in suspension $\left(\mathrm{C}_{0}=\mathrm{M} / \mathrm{V}\right)$. Combining eq. 5 and 6 ,

$$
\frac{d}{d t}\left(\frac{m}{M}\right)=\frac{1}{\tau}\left(1-\frac{m}{M}\right)
$$

where $\tau$ defines a characteristic time scale given by

$$
\tau=\frac{M}{u_{e} I \rho C_{0}} .
$$

If no sedimentation occurs, and the only change of concentration is the mass of powder deposited by EPD, for a initial time, $t=0$, the deposited mass is $\mathrm{m}(0)=0$, which leads to the solution of equation 7 ,

$$
m(t)=M\left(1-e^{-t / \tau}\right)
$$

This solution to the EPD kinetics was first proposed by Sarkar et al. [2] and is widely accepted in the literature. It reduces to the Hamaker model for short times [1] and also predicts deviations from linearity occurring when EPD is developed under constant-voltage conditions [2-4].

Biesheuvel et al. [5] described a theory of cast formation during EPD, based on the Hamaker and Avgustinik [6] equations for planar and cylindrical geometries, respectively. This theory describes the bulk effect of the particles motion in the transport phenomena near the deposition electrode, considering a non-stirred organic suspension with a zero charge density. This theory, in agreement with Sarkar et al. model, establishes that deposition depends on the variation of particles concentration in the suspension and in the formed deposit, and points out a lack in the existing theories of EPD kinetics related to the combination of mass balance (Hamaker equation) and charge balance (Poisson equation).

There are numerical models and theoretical expressions describing the relationship between resistivity and solid content of concentrated suspensions with uncharged particles. In practice, most experimental systems are formed by charged particles, where the bulk resistivity is affected by the thickness and properties of the electric double layer or the characteristics of the polyelectrolyte adsorbed at the particles surface. In fact, the resistivity of an aqueous suspension stabilized using a polyelectrolyte directly depends on the amount of dispersant added [7-10], wich is a function of the suspension solids content [11,12].

None of the models consider possible changes of the suspension resistivity during the EPD process. The aim of this work is to formulate a new resistivity model for the deposition kinetics combining the variation of colloidal parameters during the deposition process, in particular 
concentration and resistivity. This model describes more accurately experimental results obtained for longer deposition times and for suspensions with significant changes in their electrical parameters, as those dispersed in water with a large component of electrostatic repulsion.

\section{Experimental}

Tetragonal zirconia polycrystalline $\left(\mathrm{ZrO}_{2}, 3 \mathrm{~mol} \% \mathrm{Y}_{2} \mathrm{O}_{3}\right.$, Tosoh TZ3YS, Japan) powder, with a mean particle size of $0.4 \mu \mathrm{m}$, a density of $5.81 \mathrm{~g} \mathrm{~cm}^{-3}$, and a surface area of $6.7 \mathrm{~m}^{2} \mathrm{~g}^{-1}$, was used. Aqueous stabilized suspensions were prepared to a concentration of $52 \mathrm{~kg} \mathrm{~m}^{3}$ ( $5 \mathrm{wt} \%$ ), adding 0.6 $\mathrm{wt} \%$ (on a dry solid basis) of a commercial carbonic acid-based polyelectrolyte (Dolapix CE64, Zschimmer-Schwarz, Germany). Suspensions were homogenized using a high-shear mixer (Silverson L2R, UK) for 3 min.

Prepared suspensions had an electrical conductivity of $115 \mu \mathrm{S} \mathrm{cm}^{-1}$, and $\mathrm{pH} 7.9 \pm 0.1$. The electrophoretic mobility of Y-TZP suspensions was determined using a $0.04 \mathrm{~N} \mathrm{KOH}$ solution as inert electrolyte. Electrophoretic mobility measurements were performed using the microelectrophoresis technique (Zeta-meter 3.0+, Zetameter, USA) and the conductivity with a digital conductimeter (LF320 WTW, Germany).

The electrophoresis cell had a planar geometry. The work electrodes were nickel foils with an average dipping area of $9.81 \mathrm{~cm}^{2}$, and a Pt foil was used as counter electrode. EPD kinetics was determined applying a constant current density of $2.0 \mathrm{~mA} \mathrm{~cm}{ }^{-2}$ for deposition times ranging from 60 $\mathrm{s}$ up to $5,100 \mathrm{~s} .200 \mathrm{~g}$ of fresh suspension was used for each test, resulting that a total powder mass of $10 \mathrm{~g}$ could be deposited. This amount of suspension was considered enough to isolate and study the effects of any change of the suspension properties on the deposition kinetics in a reasonable time. The suspensions were stirred during the deposition tests. Conductivity and $\mathrm{pH}$ were measured after each EPD test. The effect of $\mathrm{pH}$ variations on the electrophoretic mobility of the suspensions was also estimated. The deposited mass was determined by weighting using an analytical balance.

\section{Results and discussion}

Y-TZP stable aqueous suspensions were dispersed with polyelectrolyte, in order to study the EPD kinetics as a function of electrical and colloidal parameters [7]. Table 1 summarizes the properties of as-prepared Y-TZP suspensions and the electrical conditions selected for EPD.

Table 1. Starting EPD conditions

\begin{tabular}{|l|l|}
\hline Powder mass, M & $10 \mathrm{~g}$ \\
Powder concentration, $\mathrm{C}_{0}$ & $0.052 \mathrm{~g} / \mathrm{cm}^{3}(=5 \mathrm{wt} . \%,=0.85 \mathrm{vol} . \%)$ \\
Suspension electrophoretic mobility, $\mathrm{u}_{\mathrm{e}}$ & $3.35 \times 10^{-4} \mathrm{~cm}^{2} / \mathrm{sV}$ \\
Suspension resistivity, $\rho_{0}$ & $8.7 \times 10^{3} \Omega \mathrm{cm}$ \\
Electric current intensity, I & $0.02 \mathrm{~A}$ \\
Deposition surface, $\mathrm{S}$ & $6.25 \mathrm{~cm}^{2}$ \\
\hline
\end{tabular}

After each experiment the conductivity and the $\mathrm{pH}$ were measured and the decrease of solid concentration was evaluated from the amount of powder deposited. Fig.1 shows the relationship between the suspension resistivity, the variation of solids concentration and $\mathrm{pH}$. The deposition of charged particles leads to increasing resistivity thus demonstrating the importance of the polyelectrolyte in the dispersion. The variation of resistivity with $\mathrm{pH}$ depends on the variation of ionic concentration due to water electrolysis during EPD. 


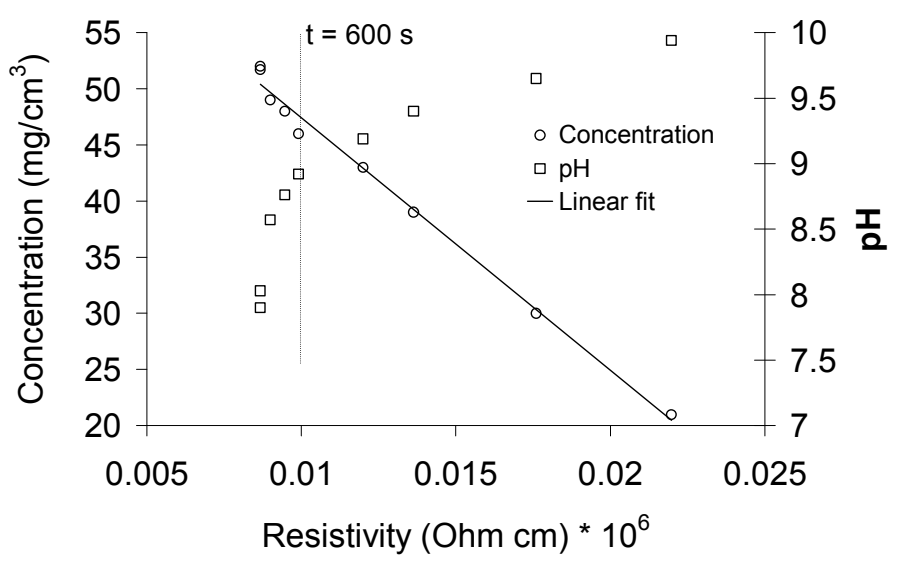

Fig. 1. Evolution of solids loading and $\mathrm{pH}$ during EPD as a function of the resistivity.

For EPD tests shorter than $600 \mathrm{~s}$ the ionic concentration changes by one order of magnitude $(\Delta \mathrm{pH}=1)$ but the suspension resistivity during maintains constant. Oppositely, the plot shows that resistivity significantly increases as solids concentration decreases. A linear relation might describe the dependence of resistivity with solids content, as follows:

$\rho=\rho_{\infty}-\left(\rho_{\infty}-\rho_{0}\right) \frac{C}{C_{0}}$

where, $\rho_{0}$ is the initial resistivity of the suspension and $\rho_{\infty}$ is the resistivity at infinite time, when $C$ $=0$. Substituting Eqs. 6 and 10 in Eq. 5, a differential equation is obtained, with the form

$\frac{d}{d t}\left(\frac{m}{M}\right)==\frac{1}{\tau_{0}}\left(1-\frac{m}{M}\right)\left(1+\left(\frac{\rho_{\infty}}{\rho_{0}}-1\right) \frac{m}{M}\right)$

where the characteristic deposition time, $\tau_{0}$, is defined by Eq. 8 with $\rho=\rho_{0}$. The solution of this new equation with the same initial condition as in equation $7, \mathrm{~m}(0)=0$, is

$$
m(t)=M\left(1-\frac{1}{1+\frac{\rho_{0}}{\rho_{\infty}}\left(e^{t / \tau_{\infty}}-1\right)}\right) .
$$

where the characteristic deposition time, $\tau_{\infty}$, is defined as in equation 8 , with $\rho=\rho_{\infty}$.

Notice that if $\rho_{\infty}=\rho_{0}$ (and hence $\rho=\rho_{\infty}$ constant) the solution of this model reduces to Sarkar $\&$ Nicholson model. If $\mathrm{t}<<\tau_{\infty}$, this solution becomes that of the linear model proposed by Hamaker. So, Eq. 12 subsumes previous models of EPD kinetics.

According to the solution given in Eq. 12, it is evident that for long deposition times, $t \rightarrow \infty$, the deposited mass $\mathrm{m}(\mathrm{t}) \rightarrow \mathrm{M}$, but the qualitative behavior of the curve $\mathrm{m}(\mathrm{t})$ deserves some discussion. Eq. 11 shows that

$$
\frac{d}{d t}\left(\frac{m}{M}\right)>0
$$

whatever is the amount of deposited mass $(0 \leq \mathrm{m} / \mathrm{M}<1)$, so that the curve $m(t)$ in Eq. 12 always increases.

Differentiating again Eq. 11, using the same equation to eliminate the derivate leads to 


$$
\frac{d^{2}}{d t^{2}}\left(\frac{m}{M}\right)=\frac{1}{\tau_{0}{ }^{2}}\left(1-\frac{m}{M}\right)\left(1+\left(\frac{\rho_{\infty}}{\rho_{0}}-1\right) \frac{m}{M}\right)\left(\frac{\rho_{\infty}}{\rho_{0}}-2-2\left(\frac{\rho_{\infty}}{\rho_{0}}-1\right) \frac{m}{M}\right) .
$$

The first three factors are positive because $\rho_{\infty}>\rho_{0}$. If $\rho_{\infty} / \rho_{0} \leq 2$, which includes the Sarkar $\&$ Nicholson model in which $\rho_{\infty} / \rho_{0}=1$, the last factor will be negative and hence, $\mathrm{m}(\mathrm{t})$ will rise with a continuously decreasing slope, as in a typical saturation curve. Contrarily, if $\rho_{\infty} / \rho_{0} \geq 2$, the second derivate (Eq. 14) will be positive as long as

$0 \leq \frac{m}{M}<\frac{\rho_{\infty}-2 \rho_{0}}{2\left(\rho_{\infty}-\rho_{0}\right)}$

and will become negative above this value, thus meaning that initially the curve $\mathrm{m}(\mathrm{t})$ increases with a growing slope, promoting a saturation S-shaped curve.

Translating this to deposition time using Eq. 12, the growing of slope takes place within the range $0 \leq \mathrm{t}<\mathrm{T}$, where

$T=\tau_{\infty} \ln \left(\frac{\rho_{\infty}}{\rho_{0}}-1\right)$

The growth of the slope depends exclusively on the variation of the resistivity, and increases as $\rho_{\infty} / \rho_{0}$ increases. The shape of the $\mathrm{m}(\mathrm{t})$ curve is not affected by any other parameter. This S-shaped behaviour has already been reported in the literature for long time EPD tests, especially for aqueous suspensions, although it also appears in non-aqueous media when deposition time is long enough [13-17].

Fig. 2 shows the evolution of weight per unit area with deposition time for studied Y-TZP aqueous suspensions, the open points corresponding to experimental values. The slope of the curve changes after the $600 \mathrm{~s}$ test, from which deposition accelerates. Later, after several deposition tests, the deposition rate starts to decrease. In this particular example, this happens after a deposition time of 3,000 s, for which $73.8 \mathrm{wt} . \%$ of the starting mass of powder has deposited. The plot clearly reveals that the evolution of weight per unit area with time follows a S-shaped trend. This tendency is confirmed for tests as long as 5,100 s, where $90 \mathrm{wt} . \%$ of the initial powder is deposited. Heavier deposits cannot be obtained because gravity makes them fall down.

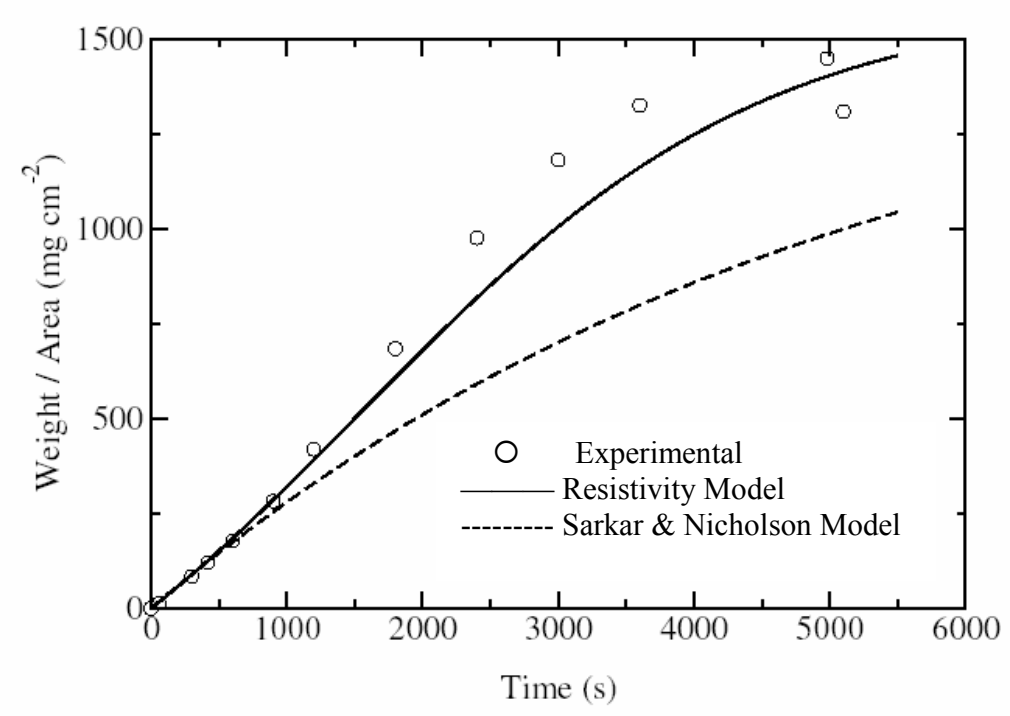

Fig. 2. EPD kinetics of a zirconia suspension. 
The Sarkar \& Nicholson model fits well the experimental values at low deposition times, but it cannot fit values above $600 \mathrm{~s}$ because the increase of the deposition rate is not captured by that model. Nevertheless, fitting Eq. 8 results in a characteristic time of 3,300 s. Experimental data show that for a deposition time of 3,000 s the solid content decreases and the deposition rate starts to decrease exponentially, as predicted by Eq. 9 .

The solid line in Fig. 2 plots the EPD kinetics according to Eq. 12, the model proposed in this work. This model approaches the experimental results for short deposition times, as the Sarkar \& Nicholson model does, but it is also capable to predict the increase of deposition rate observed after $600 \mathrm{~s}$. In fact, considering $\rho_{\infty}=23.2 \times 10^{-3} \Omega \mathrm{cm}$, the resistivity measured experimentally after a deposition time of 4,980 s, when a $90 \%$ of initial Y-TZP powder has been deposited, the deposition time during which the slope of $\mathrm{m}(\mathrm{t})$ increases (parameter $\mathrm{T}$ in eq. 16), is $630 \mathrm{~s}$, in good agreement with experimental data [7].

Hence, the proposed resistivity model is suitable for predicting the general shape of the EPD kinetics, although results are subjected to some experimental errors, especially for longer times. We have attempted to introduce corrections for such deviations by considering the influence of a possible variation of the electrophoretic mobility during EPD, but no significant improvements are achieved. As a matter of fact, other factors need to be studied in depth, one of the most important being the increase of the area available for deposition when a heavier film is obtained. In this case the conduction surface should be largely different from deposition surface, involving a new parameter that varies with time in Eq. 2.

\section{Conclusions}

Summarizing, a resistivity model has been formulated to explain the S-shaped deposition kinetics observed in long EPD tests. The model predicts a S-shape variation of mass per unit area with deposition time, with a first step in which the deposition rate increases, as a consequence of resistivity changes, followed by a decreasing slope associated to the lose of powder concentration. This model contains those of Hamaker and Sarkar \& Nicholson as particular cases, for short deposition times the former and for long deposition times and constant resistivity the latter.

\section{Acknowledgements}

This work has been supported by CICYT, Spain (MAT2003-0836). Dr. Ferrari acknowledges CSIC and European Social Foundation for the concession of a grant.

\section{References}

1. H.C. Hamaker: Trans. Faraday Soc. Vol. 36 (1940) p. 279

2. P. Sarkar and P.S. Nicholson: J. Am. Ceram. Soc. Vol. 79 (1996) p. 1897

3. Z. Zhang, Y. Huang and Z. Jiang: J. Am. Ceram. Soc. Vol. 77 (1994) p. 1946

4. M. González-Cuenca, P.M. Biesheuvel and H. Verweij: AIChE J. Vol. 626 (2000) p. 626

5. P.M. Biesheuvel and H. Verweij: J. Am. Ceram. Soc. Vol. 82 (1999) p. 1451

6. A.I. Avgustinik, V.S. Vigdergauz and G.I. Zhuravlev: J. Appl. Chem. Vol. 35 (1962) p. 2090

7. B. Ferrari and R. Moreno: J. Electrochem. Soc. Vol. 147 (2000) p. 2987

8. B. Ferrari and R. Moreno: J. Eur. Ceram. Soc. Vol. 17 (1997) p. 549

9. G. Anné, K. Vanmeensel, J. Vleugels and O. Van der Biest: Colloids and Surfaces A Vol 245 (2004) p. 35

10. S.Y. Ng and A.R. Boccaccini: Mater. Sci. \& Eng. B Vol. 116 (2005) p. 208

11. B. Ferrari and R. Moreno: Mater. Res. Bull. Vol 35 (2000) p. 887

12. S. Put, J. Vleugels and O. Van der Biest: Acta Mater. Vol. 51 (2003) p. 6303

13. J.Y. Choudhary, H.S. Ray and K.N. Rai: Trans. J. Br. Ceram. Soc. Vol. 81 (1982) p.193

14. D.E. Clark, W.J. Dalzell and D.C. Foiz: Ceram. Eng. Sci. Proc. Vol. 9 (1988) p. 1111

15. J.H. Jean: Mater. Chem. Phys. Vol. 40 (1995) p. 285

16. J. Ma and W. Cheng: J. Am.Ceram.Soc. Vol. 85 (2002) p. 1735

17. Y. Wang, I. Leu, M. Hon: J. Am. Ceram. Soc. Vol. 87 (2004) p. 84 